\title{
Aspectos da leptina na anorexia nervosa: possíveis efeitos benéficos no tratamento da hiperatividade
}

\author{
Aspects of leptin in anorexia nervosa: \\ possible beneficial effects on the \\ treatment of hyperactivity
}

Silvana Soriano FRASSETTO'

Graziela Oliveira de BITENCOURT'1

Pesquisas recentes demonstram a relação direta da leptina na regulação do balanço energético e como um dos fatores envolvidos em transtornos alimentares. Com ação no sistema nervoso central, a leptina interfere na ingestão alimentar, no metabolismo da glicose, no peso corporal, na produção de hormônios sexuais e na atividade física. As pesquisas realizadas tanto em seres humanos como em animais demonstram que a queda nos níveis de leptina está relacionada aos sintomas apresentados na anorexia nervosa: a baixa ingestão alimentar, a perda excessiva de peso corporal, a amenorréia e a hiperatividade. Assim, o grau de hipoleptinemia não é apenas uma forte indicação de baixa reserva de tecido adiposo, mas também de severa desordem, sendo que os níveis de leptina podem ser utilizados para avaliar melhor a gravidade da doença. Pesquisas estão sendo realizadas com o objetivo de discutir a possibilidade de utilização da leptina como coadjuvante no tratamento de pacientes com anorexia nervosa para a diminuição da hiperatividade. Acredita-se que o tratamento com leptina associado à medicação e à psicoterapia, poderia ser benéfico em pacientes anoréxicas extremamente ativas, deixando-as mais suscetíveis ao tratamento adicional. Uma realimentação suficiente, a medicação, a psicoterapia e um ambiente acolhedor durante o tratamento com leptina devem ser assegurados. Assim, este artigo tem como objetivo discorrer sobre a leptina e aspectos relacionados à anorexia, e discutir como esta informação pode ser importante na avaliação clínica de pacientes com este transtorno alimentar.

Termos de indexação: Anorexia nervosa. Hipercinese. Hormônios. Leptina. Tecido adiposo.

A B S T R A C T

Recent studies have demonstrated a direct relationship between leptin and the regulation of energy balance, and as a factor involved in eating disorders. Acting on the central nervous system, leptin affects food intake,

\footnotetext{
${ }^{1}$ Universidade Luterana do Brasil, Curso de Biologia. Av. Farroupilha, 8001, 92425-900, Canoas, RS, Brasil. Correspondência para/Correspondence to: S.S. FRASSETTO.E-mail:<vana@terra.com.br>.
} 
740 | S.S. FRASSETTO \& G.O. BITENCOURT

glucose metabolism, body weight, the production of sexual hormones, and physical activity. Studies with humans and animals indicate that low leptin levels are related to symptoms of anorexia nervosa: low food intake, excessive loss of body weight, amenorrhea and hyperactivity. Thus, the degree of hypoleptinemia is not only a strong indication of low reserves of adipose tissue, but also of a severe disorder, and leptin levels can be used to better evaluate the seriousness of the disease. Research has aimed to study the use of leptin in the treatment of patients with $A N$, and positive results have been obtained in the reduction of hyperactivity. It is possible that leptin treatment associated with medication and psychotherapy can be beneficial for extremely active anorexia nervosa patients, making them more susceptible to further treatment. Adequate food intake, medication, psychotherapy, and a comfortable environment during leptin treatment should be ensured. Thus, this article aims to describe leptin and aspects related to anorexia, and discuss how this information can be important in the clinical evaluation of patients with this eating disorder.

Indexing terms: Anorexia nervosa. Hyperkinesis. Hormones. Leptin. Adipose tissue.

\section{N T R O D U ÇÃ O}

A leptina é um hormônio secretado pelos adipócitos descoberto em 1994, oriundo da clonagem do gene ob e da codificação de sua proteína, que tem sua origem etimológica da palavra grega leptos (magro). Participa na regulação de vários sistemas, incluindo o sistema reprodutivo, imune, respiratório, hematopoiético e ósseo, além do seu papel no balanço energético, via sistema nervoso central ${ }^{1,2}$. Em relação ao balanço energético, atua primeiramente no Núcleo Hipotalâmico Arqueado (NHA), estimulando a expressão de neurotransmissores e hormônios ligados ao mecanismo de inibição da ingestão alimentar e do aumento do gasto energético total, via ativação do sistema nervoso simpático. Postula-se que a leptina estimule a atividade de neurônios produtores de Pró-opiomelanocorticotropina (POMC), do fator de transcrição Cocaína-Anfetamina dependente (CART) e de serotonina, com efeitos anorexígenos ${ }^{3}$. Por outro lado, inibe a expressão do Neuro-peptídeo Y (NPY) e do Agouti Related Peptide (AgRP), considerados orexígenos e redutores do gasto energético ${ }^{4,5}$.

Os níveis séricos de leptina são influenciados pela adiposidade, pela ingestão alimentar e por fatores hormonais ${ }^{6}$. A adiposidade tem sido descrita como o principal fator determinante da leptinemia, correlacionada em estudos à massa total de gordura e ao Índice de Massa Corporal $(\mathrm{IMC})^{4}$, sendo que o tecido adiposo subcutâneo tem maior produção e secreção de leptina ${ }^{6}$. Em relação à ingestão alimentar, menores concen- trações de leptina são obtidas após um jejum de 12 horas? $^{7}$.

Associada à ingestão alimentar, a insulina é um hormônio regulador da secreção de leptina pelo tecido adiposo ${ }^{8}$. Em cultura de adipócitos, a secreção de leptina depende do efeito da insulina sobre o transporte e o metabolismo da glicose nos mesmos ${ }^{9}$. Por outro lado, o cortisol, quando aumentado em restrições alimentares, inibe a secreção diurna de leptina via redução da sensibilidade insulínica, diminuindo o efeito da insulina no metabolismo da glicose no tecido adiposo ${ }^{10}$.

Assim, fatores endócrinos, a ingestão alimentar e a adiposidade interferem na leptinemia em estados de jejum e pós-prandiais. Em transtornos alimentares, como na Anorexia Nervosa (AN), sugere-se que as alterações nas concentrações de leptina contribuem para o comportamento de restrição alimentar e a hiperatividade ${ }^{11}$. Embora estudos genéticos tenham demonstrado que não há alterações específicas no gene da leptina em pacientes com AN, as alterações na leptinemia podem interferir na doença ${ }^{12}$.

Este artigo tem como objetivo discorrer sobre a leptina e aspectos relacionados à anorexia, e discutir como esta informação pode ser importante na avaliação clínica de pacientes com este transtorno alimentar. Uma vez que as ações fisiológicas da leptina incluem a redução da ingestão alimentar e o aumento do gasto energético, a discussão de possíveis efeitos da administração deste hormônio em pacientes com AN também é importante. Assim, as evidências sugerem que 
precauções devem ser tomadas em estudos iniciais com a administração de leptina em pacientes anoréxicos, para assegurar que não percam mais peso ou sofram complicações metabólicas adicionais ${ }^{13}$. Neste trabalho, serão descritos aspectos relacionados aos efeitos de alterações nas concentrações de leptina sobre o peso corporal, a função reprodutiva e os possíveis efeitos benéficos no tratamento da hiperatividade.

\section{MÉTOD OS}

Foram utilizados para a revisão artigos sobre estudos experimentais com modelos humanos ou não humanos relacionando leptina, peso corporal, função reprodutiva, hiperatividade e AN publicados em inglês e português. Trabalhos publicados em espanhol ou em outros idiomas não foram encontrados. Foram excluídos os trabalhos disponíveis somente no formato abstracts e livros texto. As bases de dados utilizadas para a pesquisa foram Medline, PubMed, Bireme, Lilacs e SciELO, buscando-se publicações disponíveis no período de 1990 a 2008, adotando-se os termos leptin, anorexia nervosa, body weight, hyperactivity, leptina, anorexia nervosa, peso corporal e hiperatividade. Foram encontrados e analisados 40 estudos que se enquadravam no propósito da revisão.

\section{Leptina e o peso corporal na anorexia nervosa}

Segundo o DSM-IV-TR ${ }^{\text {TM }} 14$ e CID-10 ${ }^{15}$, a anorexia nervosa é um transtorno alimentar que ocorre principalmente em mulheres, e apresenta um relevante distúrbio neuroendocrinológico no eixo hipotalâmico-hipofisário-gonadal ${ }^{16}$. É caracterizada pela redução na ingestão energética, perda de peso crônica à custa de dietas rígidas, distorção da imagem corporal, amenorréia e resistência ao tratamento para ganho de peso ${ }^{17}$.

As concentrações séricas e no fluido cerebroespinhal de leptina em pacientes anoréxicas são reduzidas, em relação a indivíduos sem o transtorno ${ }^{17,18}$. Mulheres constitucionalmente magras apresentam níveis intermediários de leptina, enquanto que pacientes anoréxicas apresentam concentrações plasmáticas $70 \%$ menores $^{19}$. Esta alteração se deve à restrição alimentar com a redução de massa gorda, principalmente subcutânea, refletida também na redução do IMC. Assim, restrições na ingestão de glicídios acabam por diminuir os níveis de glicose circulante, fator que diminui a secreção de leptina. Além disso, a elevação dos níveis de cortisol também é um fator relevante na queda das concentrações de leptina, bem como a intensidade e a gravidade da doença ${ }^{9}$.

As pacientes anoréxicas em tratamento apresentam aumento na leptinemia, geralmente associado ao ganho de peso e de gordura corporal, sugerindo que a leptina pode ser um indicador do estado nutricional desses indivíduos ${ }^{20}$. Em alguns estudos, as concentrações de leptina tendem a atingir a normalidade precocemente em resposta ao tratamento de realimentação e de ganho de tecido adiposo, ou até serem maiores que as do grupo controle, contribuindo para o quadro de AN e dificultando a sustentação do peso normal 17,21. Esta hiperleptinemia, que ocorre durante a recuperação, como resultado de ganho de peso rápido, sugere que o peso corporal baixo destas pacientes é fisiologicamente defendido pelo aumento dos níveis de leptina, para que ocorra novamente a restrição alimentar. Assim, a hiperleptinemia pode provocar um estado metabólico e fisiológico de regressão ao quadro de anorexia ${ }^{22}$.

Em relação ao comportamento alimentar, outros mecanismos também podem interferir na restrição nutricional na AN. Além da leptina, alterações de $\beta$-endorfina, gastrina, somatostatina e Colecistoquinina (CCK) também são descritos na $\mathrm{AN}^{23}$. Segundo hipótese apresentada por Inuii ${ }^{24}$, o NPY e o AgRP estariam aumentados como efeito compensatório, de modo a aumentar a ingestão e o ganho de peso. Por outro lado, suas ações estariam comprometidas pelo excesso de componentes anorexigênicos, como o Hormônio Liberador de Corticotrofina (CRH), a colecistoquinina e o Fator Tumoral $\alpha$ (TNF- $\alpha$ ), indicando um desequilíbrio no circuito hipotalâmico regulador da ingestão energética. Assim, esses dados podem 
contribuir para o entendimento a respeito da dificuldade de adesão ao tratamento, tendo, além da interferência psicológica, alterações que são provenientes do próprio transtorno, dificultando o retorno ao comportamento alimentar normal.

\section{Leptina e a função reprodutora}

Na puberdade, o aumento da secreção de leptina é diretamente proporcional ao aumento da gordura corporal. As concentrações de leptina são diferentes entre os sexos, sendo superiores para as mulheres a partir da puberdade, e de forma permanente após maturação sexual. A leptina atua tanto no hipotálamo como na glândula hipófise para a secreção dos Hormônios Luteinizante (LH) e Folículo Estimulante (FSH), tendo uma função na menarca como sinal ao hipotálamo de que a reserva de gordura é suficiente para uma gestação ${ }^{25}$.

Pacientes anoréxicas apresentam redução nos hormônios sexuais, que está relacionada a concentrações baixas de leptina ${ }^{26}$. As mulheres necessitam de uma concentração de leptina duas vezes mais alta que os homens, sendo também mais resistentes aos efeitos desta. Isto poderia explicar o aumento da susceptibilidade das mulheres aos transtornos alimentares e à diminuição do peso corporal na anorexia nervosa ${ }^{27}$.

A amenorréia, sintoma clínico comum em mulheres com AN, está relacionada a menores concentrações de leptina. A redução de pelo menos $15 \%$ do tecido adiposo e a leptinemia abaixo de $2 \mathrm{ng} / \mathrm{mL}$ podem causar distúrbios menstruais $^{25}$. Mesmo após a recuperação da ingestão alimentar e do peso, as pacientes anoréxicas tratadas apresentam a função do eixo hipotalâmico-hipofisário-gonadal comprometida. Assim, devido aos níveis séricos de leptina ainda reduzidos, permanece uma ausência do padrão secretório com menores concentrações de LH e de estradiol. Em pacientes com anorexia nervosa, após o tratamento adequado, muitos meses podem decorrer até que a função ovariana seja normalizada ${ }^{28}$.

\section{Leptina e hiperatividade estudos com humanos}

Níveis elevados de atividade física têm sido descritos em pacientes anoréxicas ${ }^{29,30}$, sendo que este comportamento é estudado como sendo um sintoma clínico central da $\mathrm{AN}^{31}$. Termos diferentes têm sido colocados para denominar esta atividade elevada, como exercícios em excesso ou compulsivos, uma necessidade exagerada para atividade física, hiperatividade ou inquietação motora ${ }^{29}$. As diversas descrições de hiperatividade refletem diferentes aspectos de um ou mais comportamentos relacionados ou de características psicopatológicas ${ }^{32}$.

Tem sido descrito que fatores endócrinos estão associados à hiperatividade e à restrição alimentar na anorexia nervosa ${ }^{33}$, como a diminuição de leptina e o conseqüente aumento na secreção de $\mathrm{CRH}$, do Hormônio Adrenocorticotrófico $(\mathrm{ACTH})$ e do cortisol ${ }^{34}$. Além disso, há um aumento na secreção do hormônio do crescimento $(\mathrm{GH})^{35}$. Uma relação tem sido demonstrada entre as concentrações de leptina sérica endógena e os níveis de atividade física. Pacientes anoréxicas manifestam hiperatividade quando as concentrações de leptina sérica são baixas. Por outro lado, os níveis de atividade diminuem quando o peso corporal e os níveis de leptina aumentam ${ }^{30}$. As variações nos níveis de hiperatividade em pacientes anoréxicas se correlacionam com os níveis séricos de leptina, mas não com o seu IMC, sugerindo que a hipoleptinemia está relacionada à hiperatividade física ${ }^{30}$.

Ainda não está claro se, ou em que grau, a hiperatividade é um fenótipo específico do comportamento de pacientes com AN. Em comparação com outro modelo de hipoleptinemia, a Bulimia Nervosa (BN), pacientes com anorexia nervosa, freqüentemente relatam que uma atividade física maior precede a dieta no desenvolvimento da doença quando comparados aos pacientes com BN ${ }^{29}$. Contudo, o aumento da agitação, da ansiedade e da atividade motora tem sido descrito em mulheres que fazem dieta, como nas atletas, dançarinas e ginastas ${ }^{36}$. A idade, o sexo, a dieta e os níveis de atividade antes do início do 
comportamento de restrição alimentar devem ser levados em consideração. Assim, pacientes que tinham uma atividade física ativa antes do transtorno alimentar são mais propensos a desenvolver hiperatividade na AN aguda ${ }^{32}$. Tem sido proposto que a hiperatividade em pacientes anoréxicas reflete a ativação de um sistema filogenético em indivíduos predispostos ${ }^{29}$. Na verdade, sabe-se que quando os níveis de leptina aumentam em pacientes anoréxicas, as experiências subjetivas de inquietação motora diminuem ${ }^{37}$.

\section{Estudos experimentais com animais}

O fato de que a hipoleptinemia está relacionada ao aumento da atividade física, levou a estudos na tentativa de buscar uma ação benéfica da utilização de leptina em um modelo animal de hiperatividade induzida por um Estado de Restrição Alimentar (ERA), como sendo um modelo de anorexia nervosa ${ }^{29}$. Neste estudo, ratos que tinham acesso a uma roda de corrida desenvolveram hiperatividade quando estavam em restrição alimentar. Os níveis de atividade na roda de corrida aumentaram entre 300 a 500\% em poucos dias após a restrição energética, sendo que se o experimento não fosse interrompido, os animais, provavelmente, aumentariam a sua atividade até morrerem em 7 a 10 dias. O ERA é mais freqüente nas fêmeas jovens ${ }^{29}$.

A hipótese de que a hipoleptinemia induz o estado de restrição alimentar foi confirmada experimentalmente, pela implantação subcutânea de minibombas contendo leptina ou salina em ratos submetidos à restrição de comida ${ }^{37}$. Os ratos tratados com salina demonstraram quatro vezes mais atividade física depois de sete dias. Por outro lado, os níveis de atividade física nos animais tratados com leptina permaneceram normais. Além disso, um segundo experimento demonstrou que a leptina foi capaz de recuperar os animais que tinham desenvolvido estado de restrição alimentar, sendo que os níveis elevados de atividade física diminuíram até o normal. Este papel importante da leptina no ERA foi recentemente confirmado em outro estudo ${ }^{38}$. Assim, parece que a hipo- leptinemia pode desencadear a resposta comportamental de hiperatividade, que pode ser corrigida pelo aumento dos níveis de leptina circulantes.

Ao considerar esses dados de que o estado de restrição alimentar pode ser induzido pela hipoleptinemia, tem sido sugerido que o mesmo mecanismo poderia explicar a hiperatividade em pacientes com anorexia nervosa ${ }^{37}$. Além disso, a hipoleptinemia poderia aumentar os níveis de atividade diretamente ou indiretamente, segundo características psicopatológicas específicas. Por exemplo, os efeitos independentes da ansiedade nos níveis de atividade devem ser considerados ${ }^{21}$.

A partir dos dados em animais, tem sido sugerido que a leptina poderia ser efetiva para o tratamento de hiperatividade e da hipotermia severas em pacientes anoréxicas ${ }^{34,39}$. Assim, a redução da hiperatividade poderia ser o principal objetivo para facilitar a realimentação e o ganho de peso ${ }^{38,40}$. Por outro lado, sabe-se que as pacientes precisariam, primeiramente, retomar a ingestão alimentar, caso a leptina viesse a ser utilizada como coadjuvante no tratamento da anorexia nervosa ${ }^{38}$.

\section{O N CLUSÃO}

A leptina parece desempenhar um papel importante na AN, com participação no curso da sintomatologia. Assim, a secreção de leptina é atingida com o transtorno alimentar, sendo que a normalização de seus níveis, a recuperação do peso corporal e a melhora dos sintomas somáticos e do comportamento levam algum tempo. Portanto, o grau de hipoleptinemia não é apenas uma forte indicação de baixa reserva de tecido adiposo, mas também de severa desordem, sendo que os níveis de leptina podem ser utilizados para avaliar melhor a gravidade da doença.

Tem sido demonstrado que a leptina atua juntamente com diversos hormônios como a insulina, o cortisol e os hormônios sexuais, e se relaciona diretamente com o tecido adiposo. Além disso, a leptina tem um papel importante no controle da hiperatividade, depressão e ansiedade 
em pacientes com AN, sugerindo ser um provável agente terapêutico coadjuvante. Por outro lado, estudos adicionais devem ser realizados, uma vez que a leptina não age sozinha na manutenção da ingestão e restrição alimentar.

Em relação a perspectivas de continuidade das pesquisas nesta área, estudos de correlação entre medidas de hiperatividade e os níveis de leptina na fase aguda da anorexia nervosa e durante o processo de recuperação devem ser realizados. Assim, o IMC, medidas subjetivas e objetivas dos níveis de atividade e de inquietação interior e motora, bem como os níveis de leptina sérica devem ser analisados por um longo período em pacientes adolescentes e adultos. Desta maneira, estes estudos poderão demonstrar como a leptina está relacionada ao comportamento e à cognição durante o percurso da doença.

A partir das evidências que foram descritas nesta revisão, acredita-se que o tratamento com leptina associado à medicação e psicoterapia pode ser benéfico em pacientes anoréxicas extremamente ativas, diminuindo a hiperatividade e deixando-as mais suscetíveis ao tratamento adicional. Por outro lado, em pesquisas futuras, deve-se avaliar em que patamar a administração de leptina poderia ocorrer, sempre acompanhada de realimentação suficiente e um ambiente acoIhedor buscando a plena recuperação orgânica, psicológica e social destes indivíduos.

\section{COLABORADORES}

S.S. FRASSETTO participou da concepção do trabalho, desenvolveu a análise a revisão da literatura e participou da redação do artigo. G.O. BITENCOURT auxiliou na revisão da literatura e na redação do artigo.

\section{REFER Ê N CIAS}

1. Zhang YY, Proenca R, Maffei M, Barone M, Leopold $\mathrm{L}$, Friedman JM. Positional of the mouse obese gene and its human homolog. Nature. 1994; 372(6505):425-32.

2. Havel PJ. Mechanisms regulating leptin production: implications for control of energy balance. Am J Clin Nutr. 1999; 70(3):305-6.
3. Negrão $A B$, Licinio J. Leptina: o diálogo entre adipócitos e neurônios. Arq Bras Endocrinol Metab. 2000; 44(3):205-14.

4. Banks WA, Lebel CR. Strategies for the delivery of leptin to the CNS. J Drug Target. 2002; 10(4): 297-308.

5. Niswender KD, Schwartz MW. Insulin and leptin revisited: adiposity signals with overlapping physiology and intracellular signaling capabilities. Front Neuroendocrinol. 2003; 24(1):1-10.

6. Cnop M, Landchild MJ, Vidal J, Havel PJ, Knowels NG, Carr DR, et al. The concurrent accumulation of intra-abdominal and subcutaneous fat explains the association between insulin resistance and plasma leptin concentrations. Diabetes. 2002; 51(4):1005-15.

7. Velkoska E, Morris MJ, Burns P, Weisinger RS. Leptin reduces food intake but does not alter weight regain following food deprivation in the rat. Int J Obes. 2003; 27(1):48-54.

8. Park KG, Park KS, Kim MJ, Kim HS, Suh YS, Ahn JD, et al. Relationship between serum adiponectin and leptin concentrations and body fat distribution. Diabetes Res Clin Pract. 2004; 63(2):135-42.

9. Havel PJ. Peripheral signals conveying metabolic information to the brain: short-term and long-term regulation of food intake and energy homeostasis. Exp Biol Med. 2001; 226(11):963-77.

10. Blevins JE, Schwartz MW, Baskin DG. Peptide signals regulating food intake and energy homeostasis. Can J Physiol Pharmacol. 2002; 80(5): 396-406.

11. Schwartz MW, Woods SC, Porte DJR, Seeley RJ, Baskin DG. Central nervous system control of food intake. Nature. 2000; 404(6778):661-71.

12. Hinney A, Bornscheuer A, Depenbusch M, Mierke B, Tolle A, Middeke $K$, et al. No evidence for involvement of the leptin gene in anorexia nervosa, bulimia nervosa, underweight or early onset extreme obesity: identification of two novel mutations in the coding sequence and a novel polymorphism in the leptin gene linked upstream region. Mol Psychiatry. 1998; 3(6):539-43.

13. Hebebrand J, Muller TD, Holtkamp K, Herpertz-Dahlmann B. The role of leptin in anorexia nervosa: clinical implications. Mol Psychiatry. 2007; 12(1):23-35.

14. DSM-IV-TRTM. Manual diagnóstico e estatístico de transtornos mentais. 4a. ed. Porto Alegre: Artmed; 2002.

15. Organização Mundial de Saúde. Classificação de transtornos mentais e de comportamento da CID-10. Descrições clínicas e diretrizes diagnósticas. Porto Alegre: Artes Médicas; 1993. 
16. Eckert E, Pomeroy C, Raymond N, Kohler PF, Thuras $P$, Bowers $C Y$. Leptin in anorexia nervosa. J Clin Endocrinol Metab. 1998; 83(3):791-5.

17. Gendall KA, Kaye WH, Altemus M, McConaha CW, La Via MC. Leptin, neuropeptide $Y$, and peptide YY in long-term recovered eating disorder patients. Biol Psychiatry. 1999; 46(2):292-9.

18. Krivoza J, Papezova H, Haluzikova D, Parizkova J, Jiskra J, Kotrli Haas T, et al. Soluble leptin receptor levels in patients with anorexia nervosa. Endocrinol Res. 2002; 28(3):199-205.

19. Polito A, Jabbri A, Ferro-Luzzi A, Cuzzolaro M, Censi L, Ciarapica D, et al. Basal metabolic rate in anorexia nervosa: relation to body composition and leptin concentration. Am J Clin Nutr. 2000; 71(6): 1495-502.

20. Janeckova $R$. The role of leptin in human physiology and pathophysiology. Physiol Res. 2001; 50(5):443-59.

21. Holtkamp K, Hebebrand J, Mika C, Herr M, Heussen N, Herpertz-Dahlmann B. High serum leptin levels subsequent to weight gain predict renewed weight loss in patients with anorexia nervosa. Psychoneuroendocrinology. 2004; 29(6):791-97.

22. Hebebrand J, Blum WF, Barth N, Coners H, Englaro $P$, Juul A, et al. Leptin levels in patients with anorexia nervosa are reduced in the acute stage and elevated upon short-term weight restoration. Mol Psychiatry. 1997; 2(4):330-4.

23. Baranowka B, Radzikowska M, WasilewskaDziubinska E, Roguski K, Borowiec M. Disturbed release of gastrointestinal peptides in anorexia nervosa and in obesity. Diabetes Obes Metab. 2000; 2(2):99-103.

24. Inui A. Eating behavior in anorexia nervosa: an excess of both orexigenic and anorexigenic signaling? Mol Psychiatry. 2001; 6(6):620-4.

25. Goumenou AG, Matalliotakis IM, Koumantakis GE, Panidis DK. The role of leptin in fertility. Eur J Obstetr Ginecol Reprod Biol. 2003; 106(2):118-24.

26. Tolle V, Kaden M, Bluet-Pajot MT, Jure D, Foulon C, Bossu $C$, et al. Balance in ghrelin and leptin plasma levels in anorexia nervosa patients and constitutionally thin women. J Clin Endocrinol Metab. 2003; 88(1):109-16.

27. Licinio J, Negrão AB, Mantzoros C. Sex differences in circulating human leptin pulse amplitude: clinical implications. J Clin Endocrinal Metab. 1998; 83(11):4140-7.

28. Brambilla F, Monteleone P, Bortolotti F, Dalle Grave $R$, Todisco P, Javaro A, et al. Persistent amenorrhoea in weight-recovered anorexics: psychological and biological aspects. Psychiatry Res. 2003; 118(3): 249-57.
29. Hebebrand J, Exner C, Hebebrand K, Holtkamp C, Casper RC, Remschmidt H, et al. Hyperactivity in patients with anorexia nervosa and in semi-starved rats: evidence for a pivotal role of hypoleptinemia. Physiol Behav. 2003; 79(1):25-37.

30. Holtkamp K, Herpertz-Dahlmann B, Mika C, Heer M, Heussen N, Fichter $M$, et al. Elevated physical activity and low leptin levels co-occur in patients with anorexia nervosa. J Clin Endocrinol Metab. 2003; 88(11):5169-74.

31. Hebebrand J, Casper R, Treasure J, Schweiger U. The need to revise the diagnostic criteria for anorexia nervosa. J Neural Transm. 2004; 111(7): 827-40.

32. Davis C, Katzman DK, Kaptein S, Kirsh C, Brewer H, Kalmbach $\mathrm{K}$, et al. The prevalence of high-level exercise in the eating disorders: etiological implications. Compr Psychiatry. 1997; 38(6): 321-26.

33. Elmquist JK, Maratos-Flier E, Saper CB, Flier JS. Unraveling the central nervous system pathways underlying responses to leptin. Nat Neurosci. 1998; 1(6):445-50.

34. Müller TD, Föcker M, Holtkamp K, Herpertz-Dahlmann B, Hebebrand J. Leptin-mediated neuroendocrine alterations in anorexia nervosa: somatic and behavioral implications. Child Adolesc Psychiatric Clin N Am. 2008; 18(1):117-29.

35. Lanfranco F, Gianotti L, Destefanis S, Arvat E, Ghigo E, Camanni F. Endocrine abnormalities in anorexia nervosa. Minerva Endocrinol. 2003; 28(2):169-80.

36. Bamber D, Cockerill IM, Carroll D. The pathological status of exercise dependence. Br J Sports Med. 2000; 34(2):125-32.

37. Exner C, Hebebrand J, Remschmidt H, Wewetzer C, Ziegler A, Herpertz S, et al. Leptin suppresses semi-starvation induced hyperactivity in rats: implications for anorexia nervosa. Mol Psychiatry. 2000; 5(5):476-81.

38. Hillebrand JJ, Koeners MP, de Rijke CE, Kas MJ, Adan RA. Leptin treatment in activity-based anorexia. Biol Psychiatry. 2005; 58(2):165-71.

39. Baranowska B, Baranowska-Bik A, Bik W, Martynska $L$. The role of leptin and orexins in the dysfunction of hypothalamo-pituitary-gonadal regulation and in the mechanism of hyperactivity in patients with anorexia. Neuro Endocrinol Lett. 2008; 29(1):37-40.

40. Södersten P, Bergh C, Zandian M. Psychoneuroendocrinology of anorexia nervosa. Psychoneuroendocrinology. 2006; 31(10):1149-53.

Recebido em: 3/9/2007

Versão final reapresentada em: 11/2/2009

Aprovado em: 7/7/2009 